\title{
A Systematic Literature Review: Acrophobia Treatment with Virtual Reality
}

\author{
David Jose Giraldy ${ }^{1 *}$, Wilson Novaldo ${ }^{2}$ \\ ${ }^{1,2}$ Computer Science Department, School of Computer Science, \\ Bina Nusantara University, \\ Jakarta, Indonesia 11480 \\ david.giraldy@binus.ac.id; wilson.novaldo@binus.ac.id \\ *Correspondence: david.giraldy@binus.ac.id
}

\begin{abstract}
Virtual Reality (VR) is becoming more popular as a treatment option for many phobias. We present a method that uses virtual reality in treatment to cure acrophobia in this research. Acrophobia is an anxiety condition that occurs when a person is exposed to heights. The goal of our study is to see whether Virtual Reality technology can be used to treat acrophobia. We conducted a comprehensive literature evaluation on publications relevant to acrophobia and virtual reality that were located on Google Scholar using a keyword-based search strategy. We noticed that the majority of papers involve more than two persons as a sample and use virtual reality exposure treatment. The participants' anxiety levels decrease with each exposure, suggesting that VR-based exposure might be employed as an acrophobic alternative treatment.
\end{abstract}

Keywords: Virtual Reality; Acrophobia; Acrophobia Treatment.

\section{INTRODUCTION}

Humans are afraid of a variety of things. That is what distinguishes people and makes them so difficult to assess. Humans are uncomfortable because of their fear. Humans have a fear of heights as one of their anxieties. Acrophobia is a term used to describe excessive fear, particularly dread of heights.

Acrophobia is an intense fear of heights, according to Medical News Today. Others acquire these phobias by interactions with their surroundings, such as observation of someone who witnesses people around them being scared of heights and develops the same fear, trauma from a negative event that makes a person terrified of heights, and Special situations [1]. Acrophobia is characterized by an acute fear of heights accompanied by panic and anxiety. Physical symptoms of acrophobia include increased perspiration, nausea, shivering and trembling when confronted with heights, and dizziness [2].

Many locations in the contemporary world depend on heights, such as buildings, airlines, leisure spaces, and so on. According to the Skyscraper Center, Hong Kong, one of China's major cities, has 514 skyscrapers, with about 1400 overall. There are 107 skyscrapers in Jakarta [3]. As a result, proper therapy is critical to allowing persons with acrophobia to live peacefully.

A Acrophobia may be treated with exposure therapy, cognitive behavioral therapy (CBT), or hypnosis, among other methods. We'll focus on exposure treatment in particular. Exposure therapy is progressively exposing a person to the scenario, item, or other thing that causes their fear and assisting them in adjusting to it. It may just take a few treatment sessions, with the eventual objective of the individual addressing their fear. Virtual reality's potential advantages for treating phobias are currently being researched. Virtual reality was demonstrated to be an effective treatment for acrophobia in 2014 research. According to the study's authors, three to four sessions may be enough to alleviate the phobia. [1]

- Based on a literature study that we read, we present the results of acrophobic exposure therapy in this paper. In detail, the research questions for this study are:

- How does the use of virtual reality-based therapy affect people who have acrophobia?

- What are the benefits of adopting virtual reality as a rehabilitation tool for those who are afraid of heights?

- How can the experiment be done efficiently? 


\subsection{Literature Review}

\subsubsection{Virtual Reality}

The terms 'virtual' and 'reality' are combined to form the concept of virtual reality. Near is the definition of 'virtual' and reality is what we as humans experience.

Our senses and perceptual systems provide us with information about the world. Taste, touch, smell, sight, and hearing are the five senses we've all learnt about. However, they are merely our most visible sensory organs. Humans, on the other hand, have many more senses than this, such as a feeling of balance. We have a rich flow of information from the environment to our ideas because of these many sensory inputs, as well as some specialized sensory processing by our brains.

Our whole perception of reality is made up of sensory data and the sense-making machinery in our brains that process that data. It comes to reason that if you can trick your senses into receiving false information, your view of reality will alter as a result. You'd be shown a version of reality that isn't genuine yet seems to be so from your viewpoint. Virtual reality is a term we use to describe what we're talking about.

To summarize, virtual reality involves providing our senses with a computer-generated virtual world that we may interact with in some way.

Virtual reality is a three-dimensional, computergenerated world that can be examined and interacted with by a human in technical terms. That individual becomes a part of the virtual world or is immersed in it and may control items or conduct a sequence of actions while there. [4]

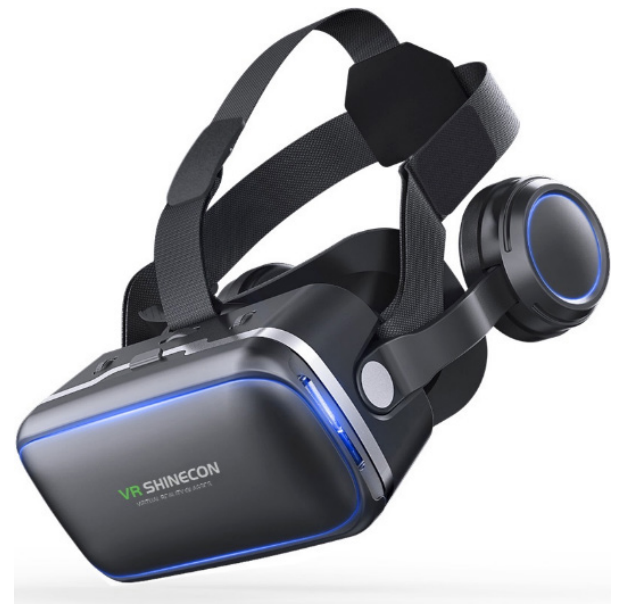

Fig. 1. Virtual Reality Headsets

Virtual reality headsets are simply computers that replace our environment with software-generated content. In order to identify how you move and monitor your activities with a virtual area, headsets use gyroscopic sensors, accelerators, and magnetometers.

The majority of VR experiences are based on these features. Field of vision and frame rate are the first two. VR creators have long been concerned with field of vision. VR must match our field of vision in order to immerse us in a new world. The reality we see around us is determined by the field of vision in your headset, as well as how closely it resembles our existing surroundings. The other visual aspect that determines how VR works is frame rate. To imitate what we see in real life, frames on a VR headset screen must move at a breakneck speed.

Spatial audio and sound effects are the second feature. Spatial audio is used in cutting-edge VR technology to recreate the type of distinctive aural environment we'd expect in the actual world. The current VR headsets employ spatial audio to communicate the direction we need to turn in and to provide a feeling of "reality" while traveling through various scenes and activities.

The third feature is head and position tracking. The degrees of freedom for head and position tracking are measured in degrees of freedom, enabling us to investigate either 6 or 3 degrees of freedom. Headsets with 6 degrees of freedom can detect your location in a room and display the direction in which your head is pointing. Eye-tracking technology may aid increase attention in virtual reality experiences and minimize nausea in certain individuals. The integration of haptic feedback sensors and other tracking technologies to include controller choices in VR may also make the environment feel more immersive. [5]

Virtual reality enables for the observation of brain function through visuals or direct recording while engaging in natural interactive activities. As a consequence, it directly answers various issues in a controlled setting, which is impossible to do while observing 'real-life' findings.

VR has been created as a post-traumatic stress disorder rehabilitation aid in psychology. Through virtual reality exposure treatment, one person will re-enact a terrible incident. It was also used to treat melancholy, anxiety, and phobias. Virtual reality technology may create a pleasant atmosphere for patients to come into touch with things they are afraid of while remaining protected and safe. [6]

\subsubsection{Acrophobia}

Acrophobia is a fear of heights that is persistent and severe. In a range of scenarios involving heights, people with acrophobia will suffer fear and immediate anxiety. Standing on a bridge, peering over a precipice, being on the top level of a building, flying, and more are examples of these scenarios.

It's crucial to comprehend, diagnose, and treat acrophobia. When left untreated, acrophobia can cause considerable distress, and significantly affect a person's life through avoidance behaviors. [7]

People who suffer from acrophobia usually have poor posture control and a lot of space and motion discomfort (SMD), which is a physical sensation induced by a lack of visual or kinesthetic information. When confronted with a high-risk scenario, acrophobia may be detected by physiological symptoms such as dizziness, shortness of breath, or heart palpitations.

To date, various phobia therapies have been devised, the most recent of which blends virtual reality with exposure therapy. Virtual reality systems have the ability to give incredibly immersive experiences. As a result, introducing virtual reality into treatment seems to be a 
feasible possibility. Exposure treatment in virtual reality is advantageous because it allows a particular phobia to be treated in a virtual setting while also conserving money.

People may set themselves in a terrible atmosphere and encounter it in virtual reality. The constant utilization of virtual reality may make anxiety less sensitive, lowering the impact of the actual circumstance. VR treatment was proved to be beneficial in treating spider phobia, social phobia, and flying phobia. Similar to a pilot research that used a virtual reality headset to lower anxiety and give a pain diversion for those who are frightened of needles, which found that 94.1 percent of participants improved after using VR [8].

\section{METHOD}

Here is the strategy that Fanny Levy, Pierre Leboucher, Gilles Rautureau, and Roland Jouvent employed in their experiment, based on their publication "E-virtual reality exposure therapy in acrophobia: A pilot research."

\subsection{Participants}

Participants are people who have acrophobia and are seeking therapy at a hospital. To participate in this study, patients must meet the current Diagnostic and Statistical Manual of Mental Disorders criteria for acrophobia (DSMIV). Patients between the ages of 18 and 65 were included in the study. To prevent restricting their ability to be therapeutically effective, patients with present depression were eliminated.

\subsection{Measure and procedure}

An unstructured clinical interview was used to screen participants for significant DSM-IV illnesses like anxiety and mood disorders. They had to fill out numerous questionnaires, including the Beck Depression Inventory (BDI), the Spielberger State-Trait Anxiety Inventory (STAI form Y-A; STAI form Y-B), the Attitude Toward Height Questionnaire (ATHQ), and the Acrophobia Questionnaire (AQ).

Each participant received a training session with the therapist. The virtual reality technique was discussed throughout the class, and participants were instructed on how to operate the virtual reality equipment. Then, for testing, they are immersed in a simulated neutral world.

Here is how the experiment is conducted.

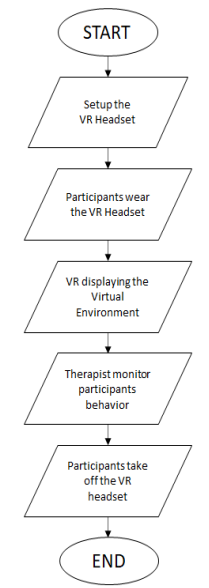

Fig. 2. Flowchart of the Experiment
Participants filled out the STAI Y-A form and evaluated their anxiety on a visual analogue scale (VAS) ranging from 0 (none) to 10 (severe) (maximum) (maximum). On the VAS, participants rated their attendance and anxiety, as well as completing a questionnaire concerning technological problems (computer, head-mounted screen, and wireless mouse) (computer, head-mounted screen, and wireless mouse). Attendance was assessed on a five-point scale ranging from 0 (none) to 10 (excellent) (maximum) (maximum). A shortened version (12 items) of the Working Alliance Inventory was used to evaluate therapeutic alliances (WAI) (WAI). One is for the patient, and the other is for the therapist. From the waiting room (five minutes of rest) until the end of the consultation, the heart rate is continuously measured using the Mio Alpha bracelet.

\subsection{Virtual reality exposure}

The virtual worlds were projected in high-resolution stereoscopic mode (3D, 1280x720) on a Sony HMZ-T1 head-mounted display. The helmet features a 3D orientation sensor that measures head and body movements during navigation. For speech communication between the therapist and the patient, a directional microphone positioned on the ceiling was used. The therapist could keep an eye on the patient's conduct while they were engaged in the virtual environment owing to a webcam.

TeamViewer 6 was used as the remote control program. Blender version 2.67 open-access software was used to generate the virtual worlds (subway stations, 24-story tower block).

\subsection{Statistical Analysis}

The findings of the e-VRET and p-VRET sessions were compared using nonparametric testing (Wilcoxon). To determine the level of anxiety generated by exposure, we calculated the differences in anxiety VAS ratings and heart rate before and after exposure.

\section{RESULT}

Two males and four women were among the participants. Table 1 shows the baseline characteristics of the population.

There were no dropouts among the participants. All of the e-VRET sessions were approved. There were no major technical issues reported. Because to software issues, none of the sessions had to be canceled or interrupted. As evidenced by the fourth presence VAS, no cybersickness (dizziness and nausea) was experienced during the sessions (negative effects). There were no negative consequences.

On the pre-exposure STAI form Y-A scores or the before-and-after difference in anxiety VAS rating, there was no significant difference between e-VRET and p-VRET. For the first three sessions, just one of the six participants' STAI form Y-A questions was missing (none for the last three). The anxiety VAS scores had no missing data.

On heart rate, there was no significant difference between e-VRET and p-VRET. 
On any of the five dimensions of the presence VAS, there was no significant difference between e-VRET and p-VRET. (Wilcoxon; immersion, $p=0.615$; realness, $p$ $=0.363$; physical presence, $p=0.532$; negative effects, $p=0.552$; therapist's assessment, $p=0.266$ ); therapist's assessment, $p=0.266$ ).

There was no statistically significant difference between the patients' assessment and the therapist's WAI ratings (Wilcoxon; $\mathrm{p}=0.381$ and $\mathrm{p}=0.381$, respectively).

Table 2 shows the results. Finally, no significant variations in anxiety, heart rate, presence, or therapeutic alliance were detected between the e-VRET and p-VRET sessions.

Table I. Participants mean (standard deviation) baseline characteristics.

\begin{tabular}{lr}
\hline Age, years & $44.5(14.2)$ \\
\hline BDI & $10.7(10.0)$ \\
STAI-Y-A & $33.9(10.0)$ \\
STAI-Y-B & $43.4(10.4)$ \\
AQ & \\
Avoidance & $19.9(7.1)$ \\
Anxiety & $74.0(12.5)$ \\
ATHQ & $45.6(9.9)$ \\
\hline
\end{tabular}

AQ: Acrophobia Questionnaire; ATHQ: Attitude Toward; Height Questionnaire; BDI: Beck Depression Inventory; STAI: Spielberger State-Trait Anxiety Inventory (state anxiety: STAI form Y-A; trait anxiety: STAI form Y-B).

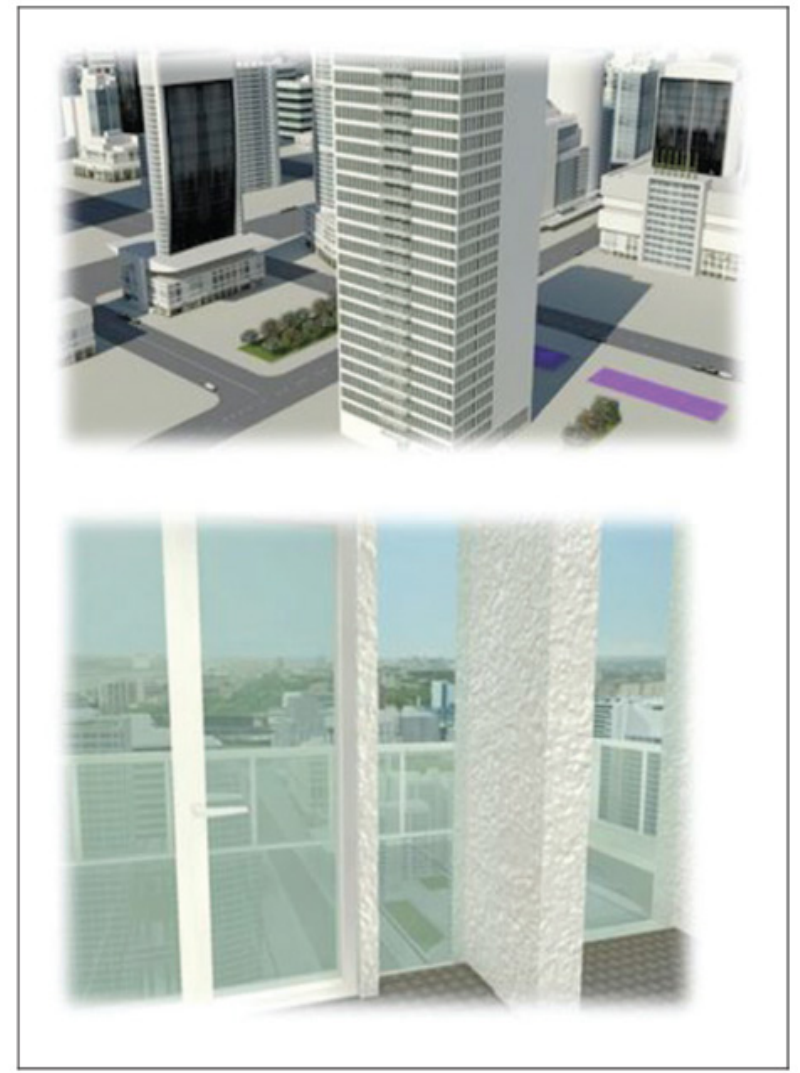

Fig. 3. Virtual world (office building)
Table 2. Comparison of mean (standard deviation) outcomes for virtual reality exposure therapy (e-VRET) in the absence of the therapist versus virtual reality exposure therapy in the presence of the therapist ( $p$-VRET).

\begin{tabular}{lccc}
\hline & e-VRET & $\boldsymbol{p}$-VRET & $\boldsymbol{p}$ \\
\hline STAI-Y-A & $34.00(8.75)$ & $32.83(7.40)$ & 0.216 \\
Difference in axiety VAS ratings & $0.99(1.80)$ & $1.59(2.50)$ & 0.256 \\
Presence & & & \\
Immersion & $5.23(3.55)$ & $5.71(3.05)$ & 0.615 \\
Realness & $5.29(3.23)$ & $5.77(3.35)$ & 0.363 \\
Physical presence & $5.95(3.16)$ & $6.00(3.19)$ & 0.532 \\
Negative effects & $2.14(3.22)$ & $2.18(3.29)$ & 0.552 \\
Therapist's evaluation & $4.96(2.48)$ & $5.65(3.06)$ & 0.266 \\
WAI & & & \\
Participant's evaluation & & & 0.786 \\
& $4.96(2.48)$ & $5.65(3.06)$ & \\
Therapist's evaluation & & & 0.168 \\
Heart rate difference & $59.83(4.49)$ & $6.67(7.37)$ & \\
\hline
\end{tabular}

STAI: Spielberger State-Trait Anxiety Inventory (state anxiety: STAI form Y-A); VAS: visual analogue scale; WAI: Working Alliance Inventory. Values of $p p$ based on Wilcoxon test.

\section{CONCLUSION}

This paper explores the application of virtual reality (VR) technology in psychology, specifically as an alternative therapy for persons who suffer from acrophobia. VR has a considerable impact on the acrophobic's anxiety level, which gradually decreases with each therapy session. The benefits of adopting virtual reality as an option for treating acrophobia include the ability to create low-cost and safe exposure therapy sessions, as well as the ability to carry out treatments in difficult and hypothetical situations. It creates a sensation of presence as well as a similar level of terror. The study had a number of flaws, the most prominent of which was the tiny number of participants. Second, all of the e-sessions were held in the hospital to avoid having to borrow equipment. Despite the fact that no serious issues arose, the feasibility of the project in the residence could not be determined. This experiment was a mock-up of what can be done via the Internet. We were able to control the settings in which the measurements (anxiety, presence, and heart rate) were taken because all of the sessions were held in the same location (hospital). Finally, we only employed one physiological anxiety measure: heart rate. In order to achieve a more precise assessment, skin conductance reactivity may have been incorporated. Finally, while we discovered no significant differences, this does not imply non-inferiority. We can't conclusively say that e-VRET and p-VRET are identical because the lack of a substantial difference could be due to a lack of power. Non-inferiority statistical testing will be used in future studies to determine equivalency between e-VRET and p-VRET. 


\section{REFERENCES}

${ }^{[1]}$ Acrophobia: Causes, symptoms, and treatment. (2020). Retrieved 10 November 2021, from https:// www.medicalnewstoday.com/articles/acrophobia\#tcauses

${ }^{[2]}$ Acrophobia, or Fear of Heights: Symptoms, Causes, and Treatment. (2019). Retrieved 10 November 2021, from https://www.healthline.com/health/ acrophobia-or-fear-of-heights-symptoms-causes-and-treatment\#symptoms

${ }^{[3]}$ Cities by Number of $150 \mathrm{~m}+$ Buildings - The Skyscraper Center. (2021). Retrieved 10 November 2021, from https://www.skyscrapercenter.com/cities.

${ }^{[4]}$ What is Virtual Reality? - Virtual Reality Society. (2020). Retrieved 23 November 2021, from https://www. vrs.org.uk/virtual-reality/what-is-virtual-reality. html.

${ }^{[5]}$ How does Virtual Reality Work?. (2021). Retrieved 23 November 2021, from https:/www.xrtoday.com/ virtual-reality/how-does-virtual-reality-work/

${ }^{[6]}$ Bohil CJ, Alicea B, Biocca FA. Virtual reality in neuroscience research and therapy. Nat Rev Neurosci. 2011;12(12):752-762.

${ }^{[7]}$ What Is Acrophobia. (2021). Retrieved 23 November 2021, from https://www.verywellhealth.com/acrophobia-5093778

${ }^{[8]}$ Acrophobia (Fear of Heights): Symptoms \& Treatment. (2021). Retrieved 23 November 2021, from https://my.clevelandclinic.org/health/diseases/21956-acrophobia-fear-of-heights

${ }^{[9]}$ Levy, F., Leboucher, P., Rautureau, G., \& Jouvent, R. (2015). E-virtual reality exposure therapy in acrophobia: A pilot study. Journal Of Telemedicine And Telecare, 22(4), 215-220. doi: $10.1177 / 1357633 \times 15598243$

${ }^{[10]}$ Rothbaum, B.O., Hodges, L., Watson, B.A., Kessler, G.D., \& Opdyke, D. (1996). Virtual reality exposure therapy in the treatment of fear of flying: A case re- port. Behaviour Research \& Therapy, 34:477-481.

${ }^{[11]}$ Botella, C., Banos, R.M., Perpina, C., Villa, H., Alcaniz, M., \& Rey, A. (1998). Virtual reality treatment of claus trophobia. Behaviour Research \& Therapy, 36:239-246.

${ }^{[12]}$ Carlin, A.S., Hoffman, H.G., \& Weghorst, S. (1997). Virtual reality and tactile augmentation in the treat ment of spider phobia: A case report. Behaviour $\operatorname{Re}$ search \& Therapy, 35:153-158.

${ }^{[13]}$ Coble, J.R., North, M.M., \& North, S.M. (1995). Effec tiveness of virtual reality environment desensitization 338 EMMELKAMP ET AL. in the treatment of agoraphobia. International Journal of Virtual Reality, 1:25-34.
${ }^{[14]}$ Emmelkamp, P.M.G., \& Felten, M. (1985). The process of exposure in vivo: Cognitive and physiological changes during treatment of acrophobia. Behaviour Research \& Therapy, 23:219-223.

${ }^{[15]}$ Storms RL, Zyda MJ. Interactions in perceived quality of auditory-visual displays. Presence: Teleoperators \& Virtual Environments 2000;9(6):557-80.

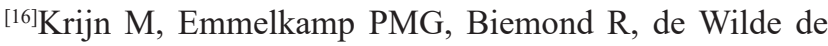
Ligny C, Schuemie MJ, van der Mast CAPG. Treatment of acrophobia in virtual reality: the role of immersion and presence. Behavior Research and Therapy 2004;42: 229-39.

${ }^{[17]}$ Krijn M, Emmelkamp PMG, Olafsson MA, Schuemie MJ, van der Mast CAPG. Do self-statements enhance the effectiveness of virtual reality exposure therapy? A comparative evaluation in acrophobia. Cyberpsychology \& Behavior 2007;10:362-70.

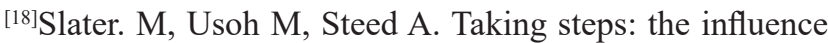
of a walking technique on presence in virtual reality. ACM Transactions on Computer-Human Interface, Special Issue on Virtual Reality Software and Technology 1995;2(3):201-19.

${ }^{[19]}$ Meehan M, Insko B, Whitton M, Boorks FP. Physiological measures of presence in stressful virtual environment. ACM Transactions on Graphics 2002;21(3):645-52.

${ }^{[20]}$ Lessiter J, Freeman J, Keogh E, Davidoff J. A cross-media presence questionnaire: the ITC sense of presence inventory. Presence: Teleoperators and Virtual Environments 2001;10(3):282-97.

${ }^{[21]}$ Ruddle RA, Lessels S. The benefits of using a walking interface to navigate virtual environments. ACM Transactions on Computer-Human Interaction 2009; 16(1):1-18.

${ }^{[22]}$ Robillard G, Bouchard S, Renaud P, Fournier T. Anxiety and presence during VR immersion: a comparative study of the reactions of phobic and nonphobic participants in therapeutic virtual environments derived from computer games. Cyberpsychology \& Behavior 2003;6(5):467-76.

${ }^{[23]}$ Choi YH, Jang DP, Ku JH, Shin MB, Kim SI. Short-term treatment of acrophobia with Virtual Reality Therapy (VRT): a case report. Cyberpsychology and Behavior 2001;4:349-454.

${ }^{[24]}$ APA. Diagnostic and statistical manual of mental disorders. Washington, DC: American Psychiatric Association; 1994.

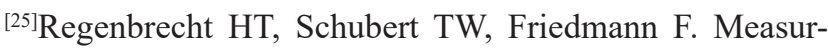
ing the sense of presence and its relations to fear of heights in virtual environments. International Journal of Human-Computer Interaction 1998;10(3):233-49.

${ }^{[26]}$ Schuemie MJ, Bruynzeel M, Drost L, Brinckman M, de Haan G, EmmelKamp PMG. Treatment of acrophobia in virtual reality: a pilot study. In: Bro- 
eckx F, Pauwels L, editors. Proceedings of Euromedia 2000, 2000. p. 271-75.

${ }^{[27]}$ Robillard G, Bouchard S, Renaud P, Fournier T. Anxiety and presence during VR immersion: a comparative study of the reactions of phobic and nonphobic participants in therapeutic virtual environments derived from computer games. Cyberpsychology \& Behavior 2003;6(5):467-76.

${ }^{[28]}$ Juan MC, Perez D. Comparison of the levels of presence and anxiety in an acrophobic environment viewed via HMD or CAVE. Presence: Teleoperators and Virtual Environments 2009;18(3):232-48.

${ }^{[29]}$ Faul F, Erdfelder E, Lang AG, Buchner A. G*Power 3: a flexible statistical power analysis program for the social, behavioral, and biomedical sciences. Behavior Research Methods 2007;39:175-91.

${ }^{[30]}$ Slater M, Garau M. The use of questionnaire data in presence studies: do not seriously likert. Presence: Teleoperators and Virtual Environments 2007;16(4):447-56.

${ }^{[31]}$ Gibson EJ, Walk RD. The visual cliff. Scientific American 1960;202:67-71. 